\title{
Functional Magnetic Resonance Imaging Demonstrates That Hypnosis Is Conscious and Voluntary
}

\section{Edoardo Casiglia',2*, Francesco Finatti2 ${ }^{2}$, Federica Gasparotti2, Maria Rosaria Stabile ${ }^{2,3}$, Micaela Mitolo ${ }^{3,4}$, Federica Albertini2,5, Antonio M. Lapenta2 ${ }^{2}$, Enrico Facco ${ }^{2,6}$, Valérie Tikhonoff ${ }^{2,7}$, Annalena Venneri ${ }^{3,8}$}

\author{
${ }^{1}$ Studium Patavinum (Formerly Department of Medicine), University of Padua, Padua, Italy \\ ${ }^{2}$ Italian Centre of Clinical and Experimental Hypnosis, Institute F. Granone, Turin, Italy \\ ${ }^{3}$ Department of Neuroimaging, Foundation Hospital San Camillo, Venice, Italy \\ ${ }^{4}$ Functional Magnetic Resonance Unit, S. Orsola-Malpighi Hospital, Bologna, Italy \\ ${ }^{5}$ School of Emergency Medicine, University of Padua, Padua, Italy \\ ${ }^{6}$ Studium Patavinum (Formerly Department of Neuroscience), University of Padua, Padua, Italy \\ ${ }^{7}$ Department of Medicine, University of Padua, Padua, Italy \\ ${ }^{8}$ Royal Hallamshire Hospital, University of Sheffield, Sheffield, UK \\ Email: *edoardo.casiglia@unipd.it
}

How to cite this paper: Casiglia, E. Finatti, F., Gasparotti, F., Stabile, M. R., Mitolo, M., Albertini, F., Lapenta, A. M., Facco, E., Tikhonoff, V., \& Venneri, A. (2018). Functional Magnetic Resonance Imaging Demonstrates That Hypnosis Is Conscious and Voluntary. Psychology, 9, 1571-1581.

https://doi.org/10.4236/psych.2018.97095

Received: June 4, 2018

Accepted: July 2, 2018

Published: July 5, 2018

Copyright $\odot 2018$ by authors and Scientific Research Publishing Inc. This work is licensed under the Creative Commons Attribution International License (CC BY 4.0).

http://creativecommons.org/licenses/by/4.0/

\begin{abstract}
Hypnosis is a condition of modified consciousness (monoideism) resulting from a mental representation able to produce psychological and physical effects. The general belief is that hypnosis is conscious and voluntary, but the practical demonstration of this hypothesis is far to be demonstrated. Twenty healthy highly hypnotizable volunteers were studied during through functional magnetic resonance imaging during a task. The task was necessary because functional magnetic resonance imaging gives no interesting results in neutral hypnosis. During the hypnotic task, the prefrontal dorso-lateral cortex, genual cortex, dorsal anterior cingulate cortex, and orbital portion of the inferior frontal convolution (i.e. the Broadmann areas 9, 25, 32 and 47) were activated. Such areas are associated to egoic consciousness and voluntary processes. The results show that the hypothesis that hypnosis is conscious and voluntary is correct.
\end{abstract}

\section{Keywords}

fMRI, Consciousness, Monoideism, Brodmann Areas, Hypnotic Focused Analgesia

\section{Introduction}

What is hypnosis. Hypnosis, a physiological modification of egoic conscious- 
ness, is a dynamic process deriving from a mental representation auto- or hetero-induced. We previously demonstrated that this mental image, active in a psychosomatic frame, produces effects not only at a psychic level (the field of application of psychotherapy), but also at a physical level, e.g. cardiovascular (Casiglia et al., 2007, 2012, 2015, 2016, 2018b; Facco et al., 2011), splanchnic (Tikhonoff et al., 2018), immunological (Wood et al., 2003), neurological (Casiglia et al., 2018a) and so on. This process, never clearly defined, has been called in different ways, such as ideomotor effect, ideoplasia, ideodynamism, and plastic monoideism (Casiglia, 2012). The more the mental image is strong and concrete, the more the hypnotic state is deep.

Is hypnotic state consciously, critically and voluntarily accepted? Although not universally shared, the prevalent belief is that hypnosis ("hypnotic trance") is consciously experienced, and hypnotism (the action of producing hypnosis) is voluntarily and critically accepted by the participant. Nevertheless, the few studies previously aimed at finding the neural processes associated with hypnotic modified consciousness were disappointing (Faymonville et al., 2000), and the need of clear demonstration that hypnosis is a conscioius and voluntary process is compelling.

The present study uses functional magnetic resonance imaging (fMRI) to verify if conscious and voluntary quality of hypnosis can be experimentally demonstrated employing the means, methods and instruments that are typical of human physiology and medical semiotics.

Why hypnosis during a task? As the imaging obtained in neutral hypnosis outside a task does not give definite information (McGeown et al., 2009; Mazzoni et al., 2013), a specific task, represented herein by hypnotic focused analgesia (HFA), was applied in the present research.

HFA was chosen as a task because it is easy to obtain in deep hypnosis and because our research staff is very expert in this field (Casiglia et al., 2007, 2015, 2016, 2018).

Aim of the study. The study described herein is aimed at clarifying whether hypnosis, when realized in the frame of a task, is characterized by activation of brain areas that are associated with consciousness and free will. The final aim of the study is to clarify if, based on brain imaging, it is plausible to state that the participants adhere to hypnosis consciously and voluntarily.

\section{Methods}

\subsection{Participants}

The study group was represented by twenty young, healthy, highly hypnotizable participants (Harvard Group Scale of Hypnotisability score $\geq 9$ ) (De Pascalis et al., 2000), represented by 5 men and 15 women aged $30.3 \pm 9.7$ years, whose educational level was $18.1 \pm 2.6$ years of schooling. They accepted to participate because of scientific curiosity and in order to offer an opportunity to clarify a hot topic. High hypnotizability was requested by the protocol, which was aimed 
at demonstrating variations of activation of brain areas in response to hypnosis. Subjects free from clautrophobia were selected, as permanence in the fMRI tunnel for at lest one hour was required.

\subsection{Safety and Ethics}

Participants were judged fit for hypnosis on the basis of anamnestic questionnaires, confidential interview with the principal investigator and, when necessary, the Minnesota Multiphasic Personality Inventory 2 Restructured Form (Locke, 2013). This procedure was aimed at screening the participants with a borderline personality more prone to develop side effects during hypnotic dissociation.

The study was approved by the Ethics Committee of the University Hospital in Padova and by that of the Fondazione Ospedale San Camillo in Venice, where fMRI scanning took place. The study was performed according to the Declaration of Helsinki for Human Research (41 ${ }^{\text {st }}$ World Medical assembly, 1990). Each subject was previously and personally informed about the aim, the meaning and the possible risks of the procedure, and was free to ask all questions to have a complete comprehension of them. All the participants gave a valid informed consent and signed a form approved by the Ethics Committee according to Italian law 675/1996 and to the law of the Veneto Region 34/2007. The participants were informed that the study had a scientific interest only, without any clinical relevance. They were also told that, once inside the fMRI scanner, they should rest during the acquisition process, listening with open eyes to the instructions given by the hypnotist through the headphones. The hypnotist did not enter the magnetic fMRI room, but was constantly in contact with the participant via an intercom and checked their conditions from another room separated by the a-magnetic windowpane. In a previous paper (Casiglia et al., 2018) we demonstrated that hypnotic induction and management is possible using headphones and radio devices.

\subsection{Protocol}

Preliminary session. All participants preliminarily underwent individual hypnotic induction through verbal suggestions. The aim of this preparatory procedure was to establish an interpersonal rapport between the operator and the participant, in order to favour a rapid and valid monoideism during the following experimental setting. The voice of an expert hypnotist guided each participant towards focusing their attention on a single idea, excluding any other external or internal stimuli. Hypnotic induction consisted of a brief enumeration coupled with suggestions of eyelid heaviness and staring at a point. The verification of hypnosis was based on some signals, such as arm levitation, the easing of facial tension, a dropped lower jaw with a slight opening of the mouth, and the slowing down of breathing rate. This phenomenological approach was sufficient to ascertain the presence of deep hypnosis. The analysis of these signals enabled the 
hypnotist to verify whether the participants were really hypnotized, and to maintain or modify this condition by means of continuous appropriate suggestions. A more accurate verification of hypnosis was performed using hypnotic focused analgesia (HFA) as a marker when the participants performing a task in the fMRI scanner during the subsequent experimental setting.

During the preliminary session, a post-hypnotic command was left to each participant in order to obtain rapid and deep hypnotic trance during the experimental setting. The aim of this command was to reduce the time needed for further inductions. To this aim, the hypnotic conditioning was given to reach hypnosis immediately when receiving the command "please, [name], relax". The effectiveness of this conditioning was tested immediately before the end of the session. The participant was then de-hypnotized and sent home.

The task model. A task model was conceived with the dual aim to commit participants to apply themselves, and to be used as a marker of goodness of deep hypnotic trance.

The task was represented by a cold pressor test (CPT) (Peckerman et al., 1991, 1994), consisting in producing a painful stimulus by immersing the participant's left hand in a plastic tub containing icy water at $0^{\circ} \mathrm{C}$ temperature. The test, not described here but reported in details elsewhere (Sevre and Rostrup, 1999; Casiglia et al., 2007), produces severe ischemic pain and a potent increase of the sympathetic discharge (Casiglia et al., 2015). CPT has already been used in the frame of experimental hypnosis by our research group (Casiglia et al., 2007, 2015, 2016) and by other Authors (Freeman et al., 2000). As water only is needed for CPT, this method is particularly fit to produce pain in fMRI setting, where cables, needles or other magnetic objects are not allowed.

Experimental session. After some days, the participants underwent the experimental setting, consisting in a sequence of fMRI scans during the task "CPT pain in HFA" and "CPT pain out of hypnosis" (control). fMRI was chosen because it is non-invasive, is advantageous in terms of temporal and spatial resolution, and does not expose the participant to radiations. It takes advantage of the atomic magnetic properties, measuring their resonance signals through magnetic fields and radio waves. In practical terms, it evaluates the hemodynamic variation in the brain areas involved in the task.

In this setting, hypnotic induction was obtained in a few seconds by means of the previously left hypnotic conditioning. This allowed inducing the participants in the scanner, when the physical presence of the hypnotist was impossible as he was outside the fMRI room and only communicated with the participant via an intercom.

Each session lasted about one hour. Once the participant was inside the scanner, their head was immobilized to minimize movements. Good comprehension of the procedure was ascertained, and headphones were placed on their ears to ensure a connection with the external environment. Experimental instructions were presented on a light-grey background display and projected via the Nordic 
Neurolab fibre optic visual presentation device mounted over the scanner head coil. Visual acuity was adjusted to individual needs. The chronological sequence of these commands was executed through the use of the program E-Prime 2. The experiment followed a box car design with an alternation of $120 \mathrm{~s}$ of "immerse" epochs and of $60 \mathrm{~s}$ of "rest" epochs.

Brain scanning took place at the Fondazione Ospedale San Camillo in Venice, Italy. Scans were acquired on a 1.5 T Philips Achieva MRI system, fitted with a Sense head coil. The scanning protocol included the acquisition of a T1-weighted structural scan, four functional scans, a T2-weighted axial scan, and a fluid attenuated inversion recovery (FLAIR) scan. Total imaging time, including localisation and structural image acquisitions, was approximately sixty minutes for each subject.

To address the experimental question, echo planar single shot $\mathrm{T} 2^{*}$ weighted MRI images (repetition time, $\mathrm{TR}=2 \mathrm{~s}$, echo time, $\mathrm{TE}=50 \mathrm{~ms}$, flip angle $=90^{\circ}$, voxel dimensions $3.28 \times 3.28 \times 5.00 \mathrm{~mm}$, field of view $240 \mathrm{~mm}$ ). 252 volumes of 30 contiguous axial slices were acquired in ascending order in each run. Each run was preceded by 30 -s dummy scans to allow the scanner to reach equilibrium, for a total scanning time of 59 minutes and $32 \mathrm{~s}$. Four runs were acquired (two in hypnosis, both for the pattern "rest" and "immerse"; two in non-hypnosis, both for the pattern "rest" and "immerse").

Analytical phase: functional image pre-processing, analysis and modelling. The Statistical Parametric Mapping 12 (SPM12) package was used in a Matlab environment on a Linux interface both for image pre-processing and statistical analysis.

The pre-processing phase included slice timing, realignment, normalization and spatial smoothing. For each voxel, slice-timing correction examined the time course and slightly moved it by interpolating with each other the points that were really detected in order to give the amount of time that would have been obtained if each voxel had been recorded exactly at the same time. The algorithm for slice-timing correction used an interpolation synchronized between time points, obtained through a Fourier's transform of the signal of each voxel. During pre-processing, all volumes of each participant were corrected by the slice-timing using the $15^{\text {th }}$ image as the reference slice (ascending acquisition). Slices were then realigned to their own mean according to the SPM12 protocol and then resliced through a $4^{\text {th }}$ degree B-spline interpolation to correct the residual movement due to signal changes. The realigned scans were normalized in a first attempt to superimpose each brain to the common template. The filter resting time (REST, part of the SPM12 toolbox) was applied after normalization but before spatial smoothing with a $6 \times 6 \times 6 \mathrm{~mm}$ width at half maximum isotropic Gaussian kernel to compensate for any residual variability after spatial normalization. Movement parameters were included as regressors in the analysis.

The analytical phase was composed of a first- and a second-level analysis. During the first-level analysis, all the runs of the participants were put together in a multiple analysis using a general linear model. Image data were high-pass 
filtered with a set of discrete cosine basis functions with a cut-off period of $200 \mathrm{~s}$. This passage generated a series of contrasts where (echo-planar imaging) EPI sequences during HFA were compared to EPI sequences without HFA.

One-sample t-tests conducted on the component images, with a threshold at $\mathrm{p}$ $<0.05$ family-wise error (FEW) cluster-level, identified the various areas. This resulted in the acquisition of $x, y, z$ coordinates in the Minnesota Neurological Institute (MNI) space using models of standard brains from the Montreal Neurological Institute; the coordinates of the significant correlations were converted in Talairach coordinates with the Matlab function mni2tal and later identified using the Talairach Daemon Client program. Differences were found comparing the pattern "immerse/rest" during HFA and during non-hypnosis. Images were presented according to the neurological convention, where right indicates the right hemisphere and left the left hemisphere.

\section{Results}

General trend. During the phase of neutral hypnosis, the parietal, frontal and limbic lobes were generically interested, with a prevalent involvement of right hemisphere. During the "CPT pain out of hypnosis" phase, an activation of the primary somatosensory areas [Brodmann areas (BA) 1, 2, and 3] was observed, demonstrating CPT really produced a perceived pain. During the task "CPT in HFA", BA 1, BA 2 and BA 3 were no more involved, demonstrating the efficacy of the task and the achievement of deep hypnosis with realization of the plastic monoideism represented by HFA (Table 1 ).

Specific trend during hypnotic task. During the task "CPT in HFA", the dorso-lateral pre-frontal cortex (BA 9), the genual cortex (BA 25), the dorsal anterior cingulate cortex (BA 32), the orbital portion of the inferior frontal (BA 47) were activated (Table 1). This was patent in the images obtained with the Talairach stereotactic system (Figure 1), where the activated BA 9, 32 and 47 were evident in the transverse and coronal projections, and the sagittal projection also put in evidence activation in BA 25. These areas were inactive when the task was performed out of hypnosis.

\section{Discussion}

A hypothesis. The mechanisms underlying hypnosis are sustained by weak theories (Mazzoni et al., 2013). Many authors acknowledge hypnosis is a physiological modification of consciousness, in other word a conscious state. Another cornerstone of the theories of hypnosis is that the participant exposes him/herself to the rapport deliberately, intentionally and voluntarily, without any coercion or subordination. As a matter of fact, it is generally assumed that hetero-hypnosis performed in the frame of a setting of hypnotism is accepted consciously and with a critical mind.

Discussion of results. The results of the present study demonstrate that the brain areas activated during hypnosis were just those supposed to be associated with consciousness and free will. 
Table 1. Brain areas activated during a hypnotic task performed in 20 highs.

\begin{tabular}{|c|c|c|c|c|c|c|c|c|}
\hline \multirow{2}{*}{ Areas } & \multirow{2}{*}{ Hemisphere } & \multirow{2}{*}{ BA } & \multirow{2}{*}{$\begin{array}{l}\text { Cluster } \\
\text { (voxels) }\end{array}$} & \multirow[b]{2}{*}{$p^{*}$} & \multirow{2}{*}{$\begin{array}{c}\mathrm{Z} \\
\text { value }\end{array}$} & \multicolumn{3}{|c|}{ Talairach coordinates } \\
\hline & & & & & & $x$ & $y$ & $z$ \\
\hline Medial frontal gyrus & left & 9 & 9980 & $<0.0001$ & 4.86 & -10 & 34 & 28 \\
\hline Cingulate gyrus (limbic lobe) & right & 32 & 9980 & $<0.0001$ & 4.09 & 10 & 32 & 28 \\
\hline Anterior cingulate cortex & right & 25 & 9980 & $<0.0001$ & 3.84 & 4 & 0 & -5 \\
\hline Subcallosus gyrus (frontal lobe) & left & 47 & 9980 & $<0.0001$ & 3.73 & -20 & 21 & -11 \\
\hline Cingulate gyrus (limbic lobe) & left & 32 & 9980 & $<0.0001$ & 3.50 & -4 & 21 & 32 \\
\hline
\end{tabular}

BA: Brodmann areas. $\ddagger$ corrected for free water excess.

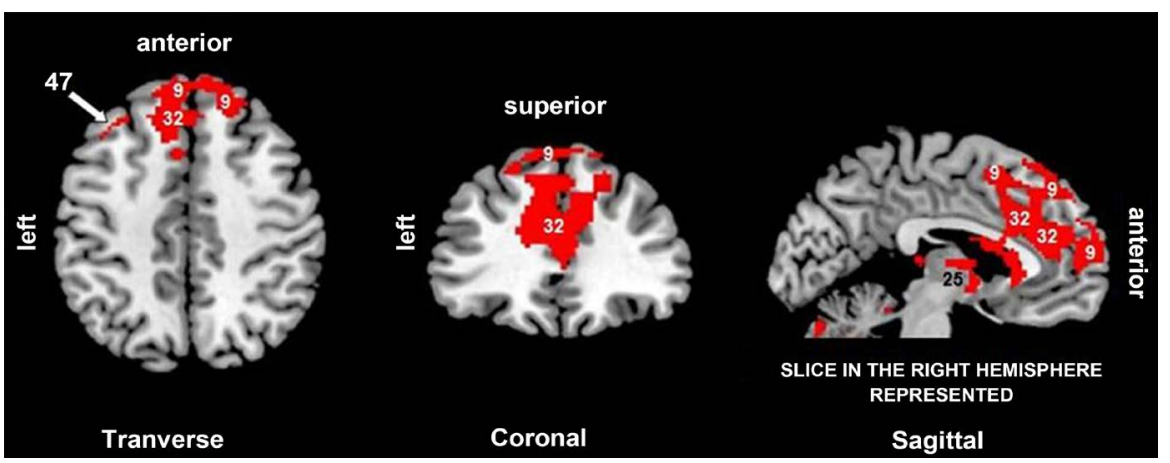

Figure 1. Functional magnetic resonance imaging of the brain of 20 highly hypnotizable volunteers in the transverse, coronal and sagittal projections. Brodmann areas that were activated during the hypnotic task are indicated by their numbers. As figure adopts the neurological convention, the sagittal projection shows a slice in the right hemisphere.

The egoic consciousness, whatever it is, is certainly non-local, not associated with the activity of "this or that" area, but probably deriving from the recursive activity of cerebral electric phenomena related to unstable neuronal connections (Casiglia et al., 2016; Solms, 2017). Nevertheless, it is irrefutable that integrity of some brain areas is necessary for the existence of consciousness. This is demonstrated by some pathological conditions in which certain areas are damaged and consciousness fails even if the sensorium is maintained (Hannawi et al., 2015), and by some experiments where de-activation of given brain areas by electrode stimulation disrupts consciousness (Koubeissi et al., 2014).

The areas that fMRI showed to be activated during our hypnotic task (BA 9, 25,32 and 47) are commonly considered necessary for egoic consciousness, and also associated with voluntary processes (Solms, 2017). BA 9 is involved in empathy (Farrow et al., 2001), in comprehension of language (Lauro et al., 2008), in taking in and processing pleasant and unpleasant scenes (Lane et al., 1997), in self-criticism (Longe et al., 2010) and in control of negative emotions (Kerestes et al., 2012), all factors implied in egoic consciousness. BA 25 (genual part of the cingulate cortex) co-ordinates a network represented by the amygdala, the insula, the hippocampus (associated to memory, which is a premise to egoic consciousness) and by some parts of the associative frontal cortex linked to self-evaluation and self-esteem (Insel, 2010). BA 32 (dorsal part of the anterior 
cingulate cortex) is involved in the attitude for representing their own and others' mental states (Mazza et al., 2008), and allows understanding of abstract expressions by extracting from speech the relevant significance going over literal meaning of words on the basis of contextual information. This is the area of interest in metaphor appreciation, which is in turn at the basis of Darwinian formation of egoic consciousness (Casiglia, 2012). BA 47 (orbital portion of the inferior frontal circumvolution) is involved in selective attention, sensorial integration with motor activity, working memory and reasoning. In Homo sapiens, the frontal/prefrontal cortex is of paramount importance in voluntary actions, to such a point that individuals in whom this network is damaged are unable to feel their own movements as a voluntary act (Nahab et al., 2017). The association between posterior cingulate, anterior insula and BA 9 seems to be particularly relevant in this context (Farrer et al., 2003; Nahab et al., 2011; Fukushima et al., 2013; Kang et al., 2015).

Strength and limitations of the study. The strength of the study is that it was based on the objective, technological evidence represented by fMRI, and confirmed hypnosis as a real and measurable state. As we are used to do (Casiglia et al., 2012), we considered hypnosis an on/off phenomenon. This could be seen as a limitation by some authors, not working in experimental setting but rather employing hypnosis in psychotherapy only, as they can be reluctant to consider the participants as "hypnotized" and "de-hypnotized". On the other hand, for researchers working with deep hypnosis in the field of neurophysiology and neuroimaging, these two states are very clearly distinguishable (Casiglia et al., 2003, 2007, 2011, 2012, 2012a, 2015, 2016, 2018, 2018a. 2018b; Facco et al., 2011; Giordano et al., 2012; Tikhonoff et al., 2018; Wood et al., 2003). Finally, different participants' hypnotizability (De Pascalis et al., 2000) or a particular echogenetic context, demonstrated in different settings (Stolarz et al., 2004; Tikhonoff et al., 2003), could have infuenced the results of the study, making confirmation in larger samples mandatory.

\section{Conclusion}

The results of the present experimental research demonstrate that: 1) hypnosis is a real and measurable state, which can be studied with the instrumental methods that are typical of human physiology; 2) hypnosis, when realized in the frame of a task, is characterized by activation of particular brain areas that are those associated with consciousness and free will, areas that are inactive when the task is performed out of hypnosis; 3 ) as a consequence, the concept that hypnosis is a physiological modification of normal consciousness and that the participant adheres to it voluntarily appears plausible. Further studies focusing on this topic using fMRI or other imaging techniques are mandatory to confirm this evidence.

\section{Acknowledgements}

This research did not receive any specific grant from funding agencies in the public, commercial, or not-for-profit sectors. 


\section{Authors' Contribution}

Edoardo Casiglia and Annalena Venneri conceived the research protocol. Edoardo Casiglia, Annalena Venneri, Francesco Finatti and Federica Albertini wrote the manuscript. Annalena Venneri, Francesco Finatti, Maria Rosaria Stabile and Micaela Mitolo analysed the fMRI data. Federica Gasparotti, Valérie Tikhonoff, Antonio M. Lapenta and Enrico Facco revised the manuscript.

\section{References}

Casiglia, E., Albertini, F., Tikhonoff, V., Gasparotti, F., Favaro, J., Finatti F., Rempelou, P., Lapenta, A. M., \& Spinella, P. (2018). Experimental Approach to the Transmission of Information in Hypnosis. Psychology, 9, 1-13. https://doi.org/10.4236/psych.2018.91001

Casiglia, E., \& Mentesana, L. (2018a). Instrumental Demonstration of Hypnotic Flaccid Paralysis of Corrugator Muscles and Its Use in Esthetic Medicine. Sleep and Hypnosis, 20, 60-66. http://www.sleepandhypnosis.org/ing/abstract.aspx?MkID=236

Casiglia, E., Rempelou, P., Tikhonoff, V., Giacomello, M., Finatti, F., Albertini, F., Favaro, J., Lapenta, A. M., \& Facco, E. (2018b). Hypnotic Focused Analgesia Obtained through Body Dysmorphism Prevents Both Pain and Its Cardiovascular Effects. Sleep and Hypnosis, 19, 89-95. http://dx.doi.org/10.5350/Sleep.Hypn.2016.18.0127

Casiglia, E., Rempelou, P., Tikhonoff, V., Guidotti, F., Pergher, V., Giordano, N., Testoni, I., \& Facco, E. (2015). Hypnotic General Anesthesia vs. Focused Analgesia in Preventing Pain and Its Cardiovascular Effects. ATINER's Conference Paper Series No. INM2015-1638.

Casiglia, E., Schiavon, L., Tikhonoff, V., Haxhi Nasto, H., Azzi, M., Rempelou, P., Giacomello, M., \& Bolzon, M. (2007). Hypnosis Prevents the Cardiovascular Response to Cold Pressor Test. American Journal of Clinical Hypnosis, 49, 255-266. https://doi.org/10.1080/00029157.2007.10524503

Casiglia, E., Tikhonoff, V., \& Facco, E. (2016). The Unconscious Experimentally Demonstrated by Means of Hypnosis. Psychology, 7, 469-479.

https://doi.org/10.4236/psych.2016.74048

Casiglia, E., Tikhonoff, V., Giordano, N., Andreatta, E., Regaldo, G., Tosello, Rossi, A. M., \& Bordin, D. (2012). Measured Outcomes with Hypnosis as an Experimental Tool in a Cardiovascular Physiology Laboratory. International Journal of Clinical and Experimental Hypnosis, 60, 241-261. https://doi.org/10.1080/00207144.2012.648078

Casiglia, E., Tikhonoff, V., Giordano, N., Regaldo, G., Facco, E., Marchetti, P., Schiff, S., Tosello, M. T., Giacomello, M., Rossi, A. M., De Lazzari, F., Palatini, P., \& Amodio, P. (2012a). Relaxation versus Fractionation as Hypnotic Deepening: Do They Differ in Physiological Changes? International Journal of Clinical and Experimental Hypnosis, 60, 338-355. https://doi.org/10.1080/00207144.2012.675297

Casiglia. E. (2012). Why Is It So Easy to Hypnotize? Contemporary Hypnosis \& Integrative Therapy, 29, 309-314.

De Pascalis, V., Russo, P., \& Marucci, F. S. (2000). Italian Norms for the Harvard Group Scale of Hypnotic Susceptibility, Form A. International Journal of Clinical and Experimental Hypnosis, 48, 44-55. https://doi.org/10.1080/00207140008410360

Facco, E., Casiglia, E., Masiero, S., Tikhonoff, V., Giacomello, M., \& Zanette, G. (2011). Effects of Hypnotic Focused Analgesia on Dental Pain Threshold. International Journal of Clinical and Experimental Hypnosis, 59, 454-468. 
https://doi.org/10.1080/00207144.2011.594749

Farrer, C., Franck, N., Georgieff, N., Frith, C. D., Decety, J., \& Jeannerod, M. (2003). Modulating the Experience of Agency: A Positron Emission Tomography Study. Neurolmage, 18, 324-333. https://doi.org/10.1016/S1053-8119(02)00041-1

Farrow, T. F., Zheng, Y., Wilkinson, I. D., Spence, S. A., Deakin, J. F., Tarrie, N., Griffiths, P. D., \& Woodruff, P. W. (2001). Investigating the Functional Anatomy of Empathy and Forgiveness. Neuroreport, 12, 2433-2438.

https://doi.org/10.1097/00001756-200108080-00029

Faymonville, M. E., Laureys, S., Degueldre, C., Del Fiore, G., Luxen, A., Franck, G., Lamy, M., \& Maquet, P. (2000). Neural Mechanisms of Antinociceptive Effects of Hypnosis. Anesthesiology, 92, 1257-1267. https://doi.org/10.1097/00000542-200005000-00013

Freeman, R., Barabasz, A., Barabasz, M., \& Warner, D. (2000). Hypnosis and Distraction Differ in Their Effects on Cold Pressor Pain. American Journal of Clinical Hypnosis, 43, 137-148. https://doi.org/10.1080/00029157.2000.10404266

Fukushima, H., Goto, Y., Maeda, T., Kato, M., \& Umeda, S. (2013). Neural Substrates for Judgment of Self-Agency in Ambiguous Situations. PLoS ONE, 8, e72267.

https://doi.org/10.1371/journal.pone.0072267

Giordano, N., Tikhonoff, V., Tosello, M. T., Lapenta, A. M., \& Casiglia, E. (2012). An Experimental Approach to Hypnotic Age Regression: Controlled Study over 10 Healthy Participants. Contemporary Hypnosis and Integrative Therapy, 29, 271-283.

Hannawi, Y., Lindquist, M. A., Caffo, B. S., Sair, H. I., \& Stevens, R. D. (2015). Resting Brain Activity in Disorders of Consciousness: A Systematic Review and Meta-Analysis. Neurology, 84, 1272-1280. https://doi.org/10.1212/WNL.0000000000001404

Insel, T. R. (2010). Faulty Circuits. Scientific American, 302, 44-51. https://doi.org/10.1038/scientificamerican0410-44

Kang, S. Y., Im, C. H., Shim, M., Nahab, F. B., Park, J., Kim, D. W., Kakareka, J., Miletta, N., \& Hallett, M. (2015). Brain Networks Responsible for Sense of Agency: An EEG Study. PLoS ONE, 10, e0135261. https://doi.org/10.1371/journal.pone.0135261

Kerestes, R., Ladouceur, C. D., Meda, S., Nathan, P. J., Blumberg, H. P., Maloney, K., Ruf, B., \& Saricicek, A. (2012). Abnormal Prefrontal Activity Subserving Attentional Control of Emotion in Remitted Depressed Patients during a Working Memory Task with Emotional Distracters. Psychological Medicine, 42, 29-40. https://doi.org/10.1017/S0033291711001097

Koubeissi, M. Z., Bartolomei, F., Beltagy, A., \& Picard, F. (2014). Electrical Stimulation of a Small Brain Area Reversibly Disrupts Consciousness. Epilepsy \& Behavior, 37, 32-35. https://doi.org/10.1016/j.yebeh.2014.05.027

Lane, R. D., Reiman, E. M., Bradley, M. M., Lang, P. J., Ahern, G. L., Davidson, R. J., \& Schwartz, G. E. (1997). Neuroanatomical Correlates of Pleasant and Unpleasant Emotion. Neuropsychologia, 35, 1437-1444. https://doi.org/10.1016/S0028-3932(97)00070-5

Lauro, L. J., Tettamanti, M., Cappa, S. F., \& Papagno, C. (2008). Idiom Comprehension: A Prefrontal Task? Cerebral Cortex, 18, 162-170. https://doi.org/10.1093/cercor/bhm042

Locke, D. E. C. (2013). Interpreting the MMPI-2-RF. The Clinical Neuropsychologist, 27, 339-341. https://doi.org/10.1080/13854046.2012.742291

Longe, O., Maratos, F. A., Gilbert, P., Evans, G., Volker, F., Rockliff, H., \& Rippon, G. (2010). Having a Word with Yourself: Neural Correlates of Self-Criticism and Self-Reassurance. Neurolmage, 49, 1849-1856.

https://doi.org/10.1016/j.neuroimage.2009.09.019 
Mazza, M., Di Michele, V., Pollice, R., Casacchia, M., \& Roncone, R. (2008). Pragmatic Language and Theory of Mind Deficits in People with Schizophrenia and Their Relatives. Psychopathology, 41, 254-263. https://doi.org/10.1159/000128324

Mazzoni, G., Venneri, A., McGeown, W. J., \& Kirsch, I. (2013). Neuroimaging Resolution of the Altered State Hypothesis. Cortex, 49, 400-410. https://doi.org/10.1016/j.cortex.2012.08.005

McGeown, W., Mazzoni, G., Venneri, A., \& Kirsch, I. (2009). Hypnotic Induction Decreases Anterior Default Mode Activity. Consciousness and Cognition, 18, 848-855. https://doi.org/10.1016/j.concog.2009.09.001

Nahab, F. B., Kundu, P., Gallea, C., Kakareka, J., Pursley, R., Pohida, T., Miletta, N., Friedman, J., \& Hallett, M. (2011). The Neural Processes Underlying Self-Agency. Cerebral Cortex, 21, 48-55. https://doi.org/10.1093/cercor/bhq059

Nahab, F. B., Kundu, P., Maurer, C., Shen, Q., \& Hallett, M. (2017). Impaired Sense of Agency in Functional Movement Disorders: An fMRI Study. PLOS ONE, 12, e0172502. https://doi.org/10.1371/journal.pone.0172502

Peckerman, A., Hurwitz, B. E., Saab, P. G., Llabre, M. M., MacCabe, P. M., \& Schneiderman, N. (1994). Stimulus Dimensions of the Cold Pressor Test and the Associated Patterns of Cardiovascular Response. Psychophysiology, 31, 282-290. https://doi.org/10.1111/j.1469-8986.1994.tb02217.x

Peckerman, A., Saab, P. G., McCabe, P. M., Skyler, J. S., Winters, R. W., Llabre, M. M., \& Schneiderman, N. (1991). Blood Pressure Reactivity and Perception of Pain during the Forhead Cold Pressor Test. Psychophysiology, 28, 485-495. https://doi.org/10.1111/j.1469-8986.1991.tb01985.x

Sevre, K., \& Rostrup, M. (1999). Blood Pressure and Heart Rate Responses to Cold Pressor Test in Patients Admitted to Hospital Due to Chest Pain. Blood Pressure, 8, 110-113. https://doi.org/10.1080/080370599438284

Solms, M. (2017). What Is "the Unconscious," and Where Is It Located in the Brain? A Neuropsychoanalytic Perspective. Annals of the New York Academy of Sciences, 1406, 90-97. https://doi.org/10.1111/nyas.13437

Stolarz, K., Staessen, J. A., Kawecka-Jaszcz, K., Brand, E., Bianchi, G., Kuznetsova, T., Tikhonoff, T., Thijs, L., Reineke, T., Babeanu, S., Casiglia, E., Fagard, R., Filipovský, J., Peleska, J., Nikitin, Y., Struijker-Boudier, H., Grodzicki, T. (2004). Genetic Variation in $C Y P 11 B 2$ and $A T 1 R$ Influences Heart Rate Variability Conditional on Sodium Excretion. Hypertension, 44, 156-162. https://doi.org/10.1161/01.HYP.0000135846.91124.a5

Tikhonoff, V., Kuznetsova, T., Stolarz, L., Bianchi, G., Casiglia, E., Kawecka-Jaszcz, K., Nikitin, Y., Tizzone, L., Wang, J. G., \& Staessen, J.A. (2003). $\beta$-Adducin Polymorphism, Blood Pressure, and Sodium Excretion in Three European Populations. American Journal of Hypertension, 16, 840-846. https://doi.org/10.1016/S0895-7061(03)00975-0

Tikhonoff, V., Senzolo, M., Lapenta, A M., Palatini, P., \& Casiglia, E. (2018). Hypnotic Hallucination of Body Heating Modifies Splanchnic Circulation: Haemodynamic and Ultrasonographic Pilot Study in Normal Volunteers. Sleep and Hypnosis, 20, 31-39. http://dx.doi.org/10.5350/Sleep.Hypn.2017.19.0132

Wood, G. J., Bughi, S., Morrison, J., Tanavoli, S., Tanavoli, S., \& Zadeh, H. H. (2003). Hypnosis, Differential Expression of Cytokines by T-Cell Subsets, and the Hypothalamo-Pituitary-Adrenal Axis. American Journal of Clinical Hypnosis, 45, 179-196. https://doi.org/10.1080/00029157.2003.10403525 